\title{
Orthopedic Correction of Class III Malocclusions during Mixed Dentition
}

\author{
Hussein N. Al-Khalifa \\ Al-Azhar University (Boys Branch), Cairo, Egypt \\ Email: drhndent@hotmail.com
}

Received 27 May 2014; revised 13 July 2014; accepted 27 July 2014

Copyright (C) 2014 by author and Scientific Research Publishing Inc.

This work is licensed under the Creative Commons Attribution International License (CC BY). http://creativecommons.org/licenses/by/4.0/

(c) (i) Open Access

\begin{abstract}
Objective: The aim of this study was to compare the effect of face mask (FM) and Maxillary Bite Block (BB) in skeletal class III patients during mixed dentition with control group of the same class during their growth. Materials \& Methods: Forty-two patients were selected based on clinical and cephalometric examination, with age ranged from 6 to 8 years according to definite criteria. Patients were classified into two groups: treated and control groups; all records were taken for every patient. Lateral cephalometric films were traced before and after treatment and analyzed. Results: Intermaxillary skeletal variables showed significant improvement in the treated group with an average increase of ANB angle (Maxilo mandibular difference angle) of $3.14^{\circ}$ and an average increase in wits appraisal $2.15 \mathrm{~mm}$. Conclusion: The study concluded that, treatment with face mask and maxillary bite block induced significant dentoskeletal changes.
\end{abstract}

\section{Keywords}

Orthopedic Correction, Class III Malocclusions, Mixed Dentition

\section{Introduction}

Orthopedic appliances are those appliances through which growth could be modified. Growth modification in skeletal Class III malocclusion can be achieved by inhibition or redirection of mandibular growth and/or stimulation of maxillary growth by traction. Extra oral appliance is an example of this orthopedic correction.

The preferred treatment for children having skeletal Class III malocclusion with retruded maxilla is the anterior movement of the maxilla where bone is added at the posterior sutures. This can be accomplished with Delaire face mask [1]-[5] that obtains anchorage from the forehead and chin or one of its modifications as, the suborbital face mask [6] that obtains its anchorage from the zygomatic bones and chin. The face mask exerts a protraction force on maxillary splints that makes the upper arch as a single unite. 
The maxillary splints may be removable [2] [4] incorporating hooks in the canine-premolar region, cemented [6] [7], or bonded [1] [8], but the later causes hygiene problems and should be avoided. An extra oral force of approximately 400 - 600 gm per side is applied by daily change of elastics that directed $30^{\circ}$ downward to pull the maxilla in a downward and forward direction, to compensate for the vertical maxillary deficiency that accompanies most cases with maxillary deficiency [7]. It is worn for 14 hours per day.

Controversy surrounds it's optimum treatment timing: effective only during early mixed dentition, under 8 years [1], effective in both age groups up to 12 years, effective in both age groups in spite of being more significant during early mixed dentition [6], and others claimed that it protrudes the maxillary bone under 8 years while only pulls the maxillary teeth forward to compensate for skeletal malocclusion in children above 9 years. The start with palatal expansion then maxillary protraction is recommended [7]. Frankel appliance is used after maxillary protraction as a retainer, to remain the facial contour and improves the profile related variables [8]. Macdonald et al. [3] recommended over correction of Class III malocclusion to compensate for post protraction growth deficiency of the maxilla.

The orthopedic and dental effects of the face mask therapy can be summarized in: it displaces the maxilla forward and rotates it in a downward and backward direction which consequently rotates the mandible also in a clockwise rotation giving rise to more convex facial profile. It moves the maxillary teeth forward while retract the lower incisors [1] [3] [8]-[10].

Treatment of class III malocclusion in growing subjects is a challenging part of contemporary orthodontic practice. Many treatment approaches can be found in the literature regarding orthopedic and orthodontic treatment in Class III malocclusion, including intraoral and extraoral appliances such as (FM) facial mask [11], (fr-3) appliance of Frankel [12], bionator [9], removable mandibular retractor [13], double-piece corrector [14], chincup [15], splints, Class III elastics, and chincup [16], and mandibular cervical headgear [17] [18].

Initial observations on the effects of posteroanterior traction of the maxillary complex in the treatment of Class III malocclusion demonstrated skeletal protraction of the maxilla, improvement of the ANB (Maxilo mandibular difference) angle, backward and downward rotation of mandible with augmentation of facial hight, and lingual tipping of the mandibular incisors [19] [20].

Clinical studies have also shown variable effects of maxillary protraction therapy on the amount of mandibular growth with the mandible rotated downwards and backwards during treatment [21]-[23]. Bite blocks (BB) have demonstrated beneficial therapeutic effects in subjects with dentoskeletal open-bite tendency, since they reduce extrusion of the posterior teeth and allow autorotation of the mandible to produce bite closure [24]. Extending these concepts [1] [25]-[27], bonded maxillary expanders were used with acrylic splints as a BB device combined with FM therapy for Class III malocclusion. Favorable outcomes of BB therapy for the control of mandibular growth direction were observed in both experimental animal and human studies [28] [29].

The purpose of this clinical study was to evaluate the therapeutic effects of a treatment protocol for Class III malocclusion consisting of a FM and a BB appliance in the maxillary arch. Active treatment effects were analyzed in consecutively treated patients.

\section{Material and Methods}

The procedures followed in the study were in accordance with the ethical standards of the responsible committee on human experimentation. The study was done on two groups of patients with total number of 24 patients with skeletal Class III malocclusion; patients were randomly selected from the outpatient clinic of orthodontic Department, Faculty of Dental Medicine, Al Azhar University Cairo, Egypt.

Patients were classified into two groups; group one, the treated group, consisted 12 patients and group two, the control group, consisted of 12 patients. Patients were selected with age ranged from 6 to 8 years and that was to give a chance for control group to have a time for their treatment after observation period that took 10 months for obtaining actual results.

All orthodontic records were taken for every patient. Lateral cephalometric films were selected from the records for analysis of pre and post treatment changes that were produced by facemask (FM) and maxillary Bite Block (BB). Delaire type facemask, in which the frontal and mental regions can be used for extraoral anchorage, Bite Block on maxillary arch with two hooks at the canine area, BB was cemented in place by Glass ionomer cement, extraoral elastics were used with average force magnitude 400 grams on each side. Elastics were directed $30^{\circ}$ inferior to the occlusal plane from mesial surface of upper canines. The elastics were worn form 12 - 
14 hours a day for 10 months. During FM treatment, a BB was used in all treated patients, with the aim to counteract any tendency to clockwise mandibular rotation. The splints were intended to control molar eruption, limit intermaxillary divergence, and prevent clockwise mandibular rotation.

The selection of patients in both of the groups was made according to the following criteria:

1) skeletal class III relationship with maxillary retrognathism, 2) ANB angle less than $0^{\circ}$, 3) All patients had no transverse discrepancy between the dental arches, 4) No craniofacial anomaly, 5) No history of pervious orthodontic treatment, 6) all patients had Class III malocclusion in the mixed dentition characterized by a Wits appraisal of $-2 \mathrm{~mm}$ or less 7) No permanent teeth were congenitally missing or extracted before or during treatment 8) Co-operative patients and parents.

In order to determine the patients' skeletal age, hand and wrist radiographs were used and analyzed. In order to evaluate changes from pretreatment (T1) to post treatment (T2), lateral cephalometric films were used to determine linear and angular measurements as in Figure 1 and Figure 2.

Lateral cephalograms were taken at the start of treatment T1 and the end of active treatment with the FM-BB protocol (T2). Analysis was used for all cephalograms examined in this study and it contained measurements from several analyses [10] [30]-[33]. Before the cephalometric analysis, the intraobserver measurement error was evaluated. Ten lateral cephalometrics, selected from various subjects in the study, were traced and measured at 2 times within a week by the same operator. The measurements at both times for each patient were analyzed with the intraclass correlation coefficient, which varied between 0.966 for the SNB angle and 0.995 for the inclination of the maxillary incisor to the Frankfort horizontal (FH) line. These values indicated a high level of intraobserver agreement. Linear measurement errors averaged $0.3 \mathrm{~mm}(\mathrm{SD}, 0.8 \mathrm{~mm})$, and angular measurement errors averaged $0.4^{\circ}\left(\mathrm{SD}, 0.6^{\circ}\right)$.

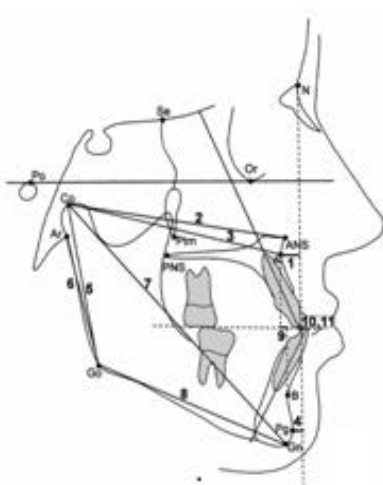

(a)

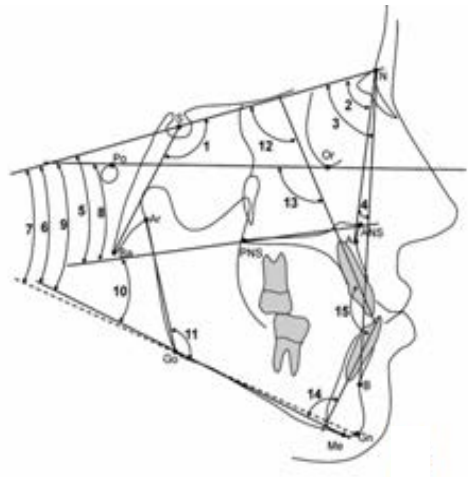

(b)

Figure 1. (a) Cephalometric Linear measurements: 1: A to N perpendicular, 2: Co-ANS, 3: Co-A 4: Pg to N perpendicular, 5: Co-Go, 6: Ar-Go, 7: Co-Gn, 8: Go-Gn, 9: Wits appraisal, 10: OJ 11-OB. (b) Cephalometric angular measurements: 1: NSBa, 2: SNA, 3: SNB, 4: ANB, 5: SN-PP, 6: SN-GoMe, 7: SN-GoGn, 8: FH-PP, 9: FH-MP, 10: PP-MP, 11: Gonial Angle, 12:U1-SN, 13: U1-FH, 14: IMPA, 15: Interincisal Angle.

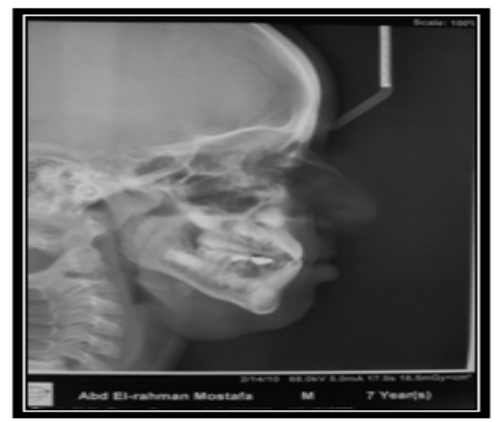

(a)

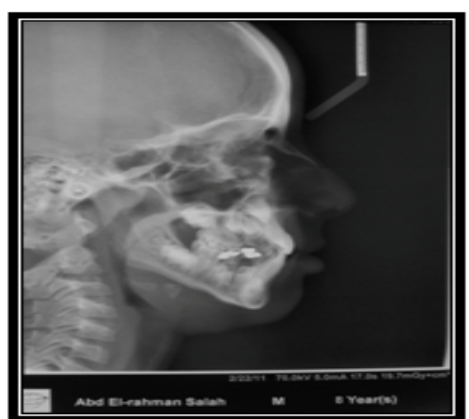

(b)

Figure 2. (a) Lateral cephalometric pre-treatment; (b) Lateral cephalometric post-treatment. 
Each cephalogram was traced at the 2 times, and linear and angular variables were measured. The cephalometric measurements used were the following): 1) cranial flexure, NSBa angle; 2) maxillary skeletal, SNA angle, A-N perpendicular (point A to a line drawn perpendicular to the FM from N), Co-ANS, and Co-A; 3) mandibular skeletal: SNB angle, Pg-N perpendicular (Pg to a line drawn perpendicular to the FM from N), Co-Go, Ar-Go, Co-Gn and Go-Gn; 4) skeletal differences: ANB angle, Wits appraisal (distance between the 2 points of intersection of the 2 perpendicular lines from points $\mathrm{A}$ and $\mathrm{B}$ to the functional occlusal plane), and maxillomandibular differential (difference between Co-A and Co-Gn); 5) vertical skeletal: SN-palatal plane(PP) angle, SN-GoMe angle, SN-GoGn angle, FH-PP angle, FH-mandibular plane (MP) angle, PP-MP angle, and gonial angle (Ar-Go-Me); and 7) dental measurements: maxillary central incisor (U1)-SN angle, U1-FH angle, IMPA angle, interincisal angle, OJ, overbite (OB). Also photographs were taken before and after treatment as in Figure 3.

\section{Results}

Several significant and non-significant differences were observed between the treated and control group at the end of treatment at $\mathrm{T} 2$.

\subsection{Treated Group}

Statistical analysis of the pretreatment and post treatment changes in the treated group showed several significant changes from T1 to T2 as shown in (Table 1). Sagittal maxillary position (SNA, A-N perpendicular), midfacial
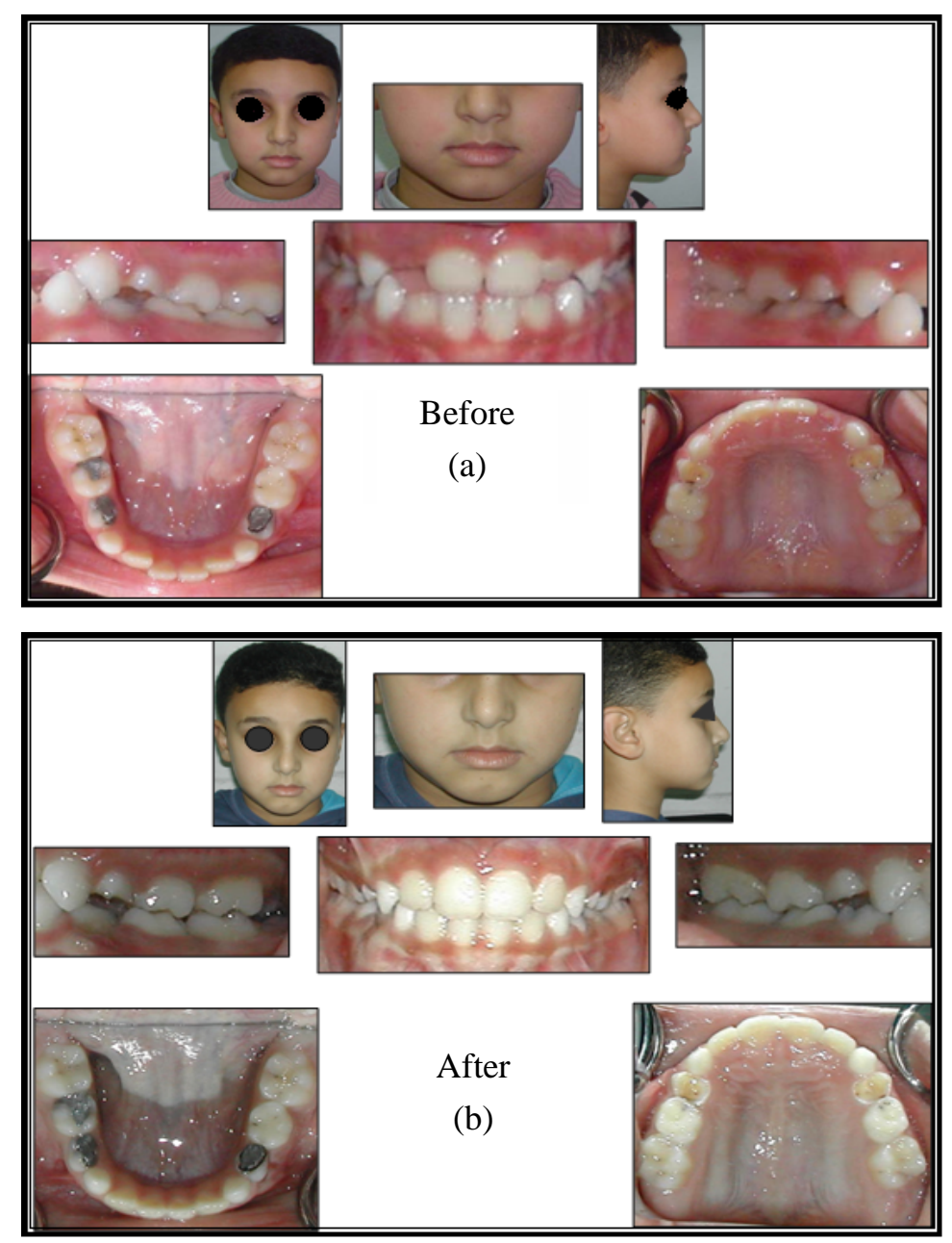

Figure 3. (a) Patient photograph before treatment; (b) Patient photograph after treatment. 
Table 1. Statistical differences between T1 and T2 in control group and in the treated group.

\begin{tabular}{|c|c|c|c|c|c|c|c|c|c|c|}
\hline & \multicolumn{4}{|c|}{ Control group } & \multirow{3}{*}{ Sig. } & \multicolumn{4}{|c|}{ Treated group } & \multirow{3}{*}{ Sig. } \\
\hline & \multicolumn{2}{|c|}{ (T1) } & \multicolumn{2}{|c|}{ (T2) } & & \multicolumn{2}{|c|}{ Pretreatment T1 } & \multicolumn{2}{|c|}{ Post treatment T2 } & \\
\hline & Mean & $\pm \mathrm{SD}$ & Mean & $\pm \mathrm{SD}$ & & Mean & $\pm \mathrm{SD}$ & Mean & $\pm \mathrm{SD}$ & \\
\hline Cranial flexure NSBa & 128.2 & 3.01 & 128.1 & 3.15 & $0.118^{\mathrm{NS}}$ & 127.06 & 2.6 & 125.8 & 3.96 & $1.03^{\mathrm{NS}}$ \\
\hline \multicolumn{11}{|l|}{ Maxillary skeletal } \\
\hline SNA & 78 & 1.51 & 78.2 & 1.42 & $-0.37^{\mathrm{NS}}$ & 78.9 & 1.22 & 81.2 & 1.37 & $-4.7^{* * * *}$ \\
\hline $\mathrm{A}-\mathrm{N} \perp(\mathrm{mm})$ & -5.06 & 1.66 & -5.13 & 1.8 & $0.105^{\mathrm{NS}}$ & -3.6 & 1.17 & -2.2 & 1.01 & $-3.65^{* *}$ \\
\hline CO-ANS (mm) & 78.8 & 1.97 & 79.8 & 1.56 & $-1.53^{\mathrm{NS}}$ & 84.4 & 0.98 & 89.2 & 1.08 & $-12.7^{* * *}$ \\
\hline CO-A (mm) & 74.4 & 2.19 & 77.26 & 2.21 & $-3.55^{* * *}$ & 74.9 & 1.16 & 80.3 & 1.11 & $-12.99^{* * * *}$ \\
\hline \multicolumn{11}{|l|}{ Mandibular skeletal } \\
\hline SNB & 80.26 & 1.27 & 80.33 & 1.54 & $-0.129^{\mathrm{NS}}$ & 81.06 & 1.27 & 79.4 & 1.18 & $3.7^{* *}$ \\
\hline Pg-N $\perp(\mathrm{mm})$ & 0.33 & 1.718 & 0.4 & 1.8 & $-0.104^{\mathrm{NS}}$ & -1.7 & 1.09 & -1.2 & 1.03 & $-1.7^{\mathrm{NS}}$ \\
\hline CO-GO (mm) & 47.06 & 2.18 & 49.13 & 1.84 & $-2.79^{* *}$ & 48.2 & 2.96 & 52.6 & 2.52 & $-4.3^{* * * *}$ \\
\hline Ar-GO (mm) & 38.26 & 1.43 & 39.26 & 1.62 & $-1.78^{\mathrm{NS}}$ & 38.2 & 2.68 & 38 & 2.32 & $0.29^{\mathrm{NS}}$ \\
\hline Co-Gn (mm) & 102.6 & 2.76 & 103.9 & 3.3 & $-1.13^{\mathrm{NS}}$ & 104 & 3.61 & 105 & 1.84 & $-1.01^{\mathrm{NS}}$ \\
\hline GO-GN (mm) & 64.4 & 1.95 & 67.9 & 1.48 & $-5.56^{* * *}$ & 65.8 & 1.64 & 65.6 & 1.34 & $0.36^{\mathrm{NS}}$ \\
\hline \multicolumn{11}{|l|}{ Skeletal difference } \\
\hline ANB & -1.26 & 0.79 & -1.16 & 0.82 & $1.12^{\mathrm{NS}}$ & -1.86 & 1.06 & 1.6 & 0.72 & $-10.6^{* * *}$ \\
\hline Wits app & -6 & 1.69 & -6.16 & 1.38 & $0.11^{\mathrm{NS}}$ & -3.6 & 1.04 & -1.2 & 1.14 & $-6.15^{* * * *}$ \\
\hline \multicolumn{11}{|l|}{ Vertical } \\
\hline SN-PP & 9.54 & 1.28 & 9.26 & 1.29 & $0.58^{\mathrm{NS}}$ & 6.8 & 1.35 & 10.8 & 1.84 & $-6.76^{* * *}$ \\
\hline SN-GO Me & 39.03 & 1.46 & 38.93 & 1.11 & $0.21^{\mathrm{NS}}$ & 35.4 & 1.18 & 36.6 & 1.63 & $-2.29^{\mathrm{NS}}$ \\
\hline SN-GO GN & 38.73 & 1.84 & 38 & 1.69 & $1.08^{\mathrm{NS}}$ & 35.7 & 1.47 & 35.2 & 1.43 & $-0.125^{\mathrm{NS}}$ \\
\hline FH-PP & 3.46 & 1.15 & 4.6 & 0.95 & $-3.09^{* *}$ & 4.5 & 1.24 & 4.1 & 1.4 & $-0.13^{\mathrm{NS}}$ \\
\hline FH-Mp & 27.43 & 2.6 & 28.8 & 2.19 & $-1.59^{\mathrm{NS}}$ & 25.8 & 1.37 & 27 & 1.35 & $-2.67^{* *}$ \\
\hline PP-Mp & 29.76 & 2.53 & 30 & 2.93 & $-0.23^{\mathrm{NS}}$ & 27.4 & 4.68 & 26.8 & 1.93 & $0.45^{\mathrm{NS}}$ \\
\hline Gonial angle & 131.4 & 2.83 & 133 & 3.18 & $-1.51^{\mathrm{NS}}$ & 126.9 & 3.21 & 126.8 & 3.18 & $0.11^{\mathrm{NS}}$ \\
\hline \multicolumn{11}{|l|}{ Dental } \\
\hline U1-SN & 100 & 3.66 & 103 & 3.66 & $-2.24^{*}$ & 102 & 2.5 & 99.2 & 2.85 & $2.85^{* *}$ \\
\hline U1-FH & 106 & 5.07 & 110 & 5.21 & $-2.12^{*}$ & 110.6 & 1.76 & 107.6 & 1.72 & $4.71^{* * *}$ \\
\hline IMPA & 89.4 & 1.96 & 90.7 & 2.36 & $-1.67^{\mathrm{NS}}$ & 91.4 & 1.72 & 88.2 & 1.48 & $5.331^{* * *}$ \\
\hline Interincisal angle & 141.6 & 3.18 & 139.5 & 1.81 & $2.18^{*}$ & 138 & 4.4 & 142.7 & 4.78 & $-2.73^{* *}$ \\
\hline Over jet & -2.26 & 1.03 & -2.76 & 1.25 & $1.19^{\mathrm{NS}}$ & -1.6 & 0.73 & 1.9 & 0.7 & $-13.4^{* * * *}$ \\
\hline Overbite & 2.32 & 0.98 & 2.26 & 0.92 & $0.15^{\mathrm{NS}}$ & 2.3 & 0.61 & 2.2 & 0.67 & $0.56^{\mathrm{NS}}$ \\
\hline
\end{tabular}

NS: non-significant, ${ }^{*}$ Statistically significant at $(\mathrm{P}<0.05)$ level, ${ }^{* * *}$ Statistically significant at $(\mathrm{P}<0.01)$ level, ${ }^{* * *}$ Statistically significant at $(\mathrm{P}<0.001)$ level. 
length (Co-A, Co-ANS), and mandibular total length (Co-Gn) were significantly greater at T2 than at T1 while (SNB) were significant smaller value at the end of treatment (T2) the values for the ANB angle and Wits appraisal showed significant improvements.

The vertical measurements showed non-significant changes except the inclination of the PP (Palatal Plane) to the cranial base and the FH (Frankfort plane) to the mandibular plane (SN-PP, FH-MP) showed significantly smaller values at T2 and the same occurred for OJ (Over Jet). A positive OJ was obtained in all subjects at T2. There were significant decreases in the axial inclination of the maxillary and mandibular teeth and greater interincisal angle in the treatment group from $\mathrm{T} 1$ to $\mathrm{T} 2$.

\subsection{Control Group}

Statistical analysis of the control group is shown in Table 1 . There were non-significant changes in the cranioflexture characteristics and Sagittal maxillary position except (Co-A) showed significant increase at T2. The total mandibular length (Co-Gn and Go-Gn) were significant increase at T2. There were non-significant changes of ANB, wits appraisal and the vertical measurements except FH-PP angle showed increase at T2. The axial inclination of upper incisors showed significant increase labially and decrease of the interincisal angle while there were non-significant changes of the over jet and overbite.

\subsection{Comparison of the Changes between Treated Versus Control Groups}

The statistical comparison of the changes from T1 and T2 in the treated and control groups (Table 1) showed many significant changes produced by active treatment changes using FM-BB appliance. The maxillary and the mandibular skeletal measurements showed significant improvement in the treated group versus control group. Intermaxillary skeletal variables showed significant improvement in the treated group with an average increase of ANB angle of $3.14^{\circ}$ and an average increase in wits appraisal $2.15 \mathrm{~mm}$. There were significant changes in the vertical values particularly FH-PP and Gonial angle showed decrease in the treated group versus control group. The over jet improved significantly from T1 to T2 in the treated group versus control group (4 mm) while the axial inclination of the upper and lower incisors decreased and the interincisal angle increased in the treated group versus control group.

\section{Discussion}

Skeletal Class III malocclusions are known to be the most difficult malocclusion types to treat among the many skeletal disorders [10]. It has been suggested that, to obtain optimum treatment results with maxillary protraction, it must be applied in the pre-pubertal period (Shanker 1996 [34]).

Face mask treatment caused a mesial movement of the basal and alveolar regions, and that anterior rotation of the maxilla and posterior rotation of the mandible occurred (Enacar and Demirhanglu, 1989) [10]. Application of face mask protraction lead to anterior rotation and displacement of the maxillary complex (Kambara, 1977 [35] and Nanda 1978 [36]).

A review of the literature reveals that early treatment with maxillary protraction appliance is effective in correcting Class III malocclusion due to maxillary retrusion (McNamara, 1987) [12].

From the results of the present study, statistical analysis of pretreatment and post treatment changes in the treated group showed several significant changes from T1 to T2 as in Table 1.

Sagittal maxillary position (SNA, A-N1), midfacial length (Co-A, Co-ANS) and mandibular length (Co-Go) were significantly greater at $\mathrm{T} 2$ than at $\mathrm{T} 1$, while ANB were significantly smaller value at the end of treatment T2. In contrast, Change et al. [37] did not reported significant improvement in maxillary position after face mask therapy in either short or long treatment.

The values for ANB angle and Wits appraisal showed significant improvement due to increased amount of forward displacement of maxilla during active treatment. This agrees with the study done by Cozza et al. [38], and with previous reports. This is due to the use of splints in maxillary arch that facilitated the forward movement of the maxilla or due to prolonged time of face mask wear (12 hours).

The present study concluded that significant maxillary protraction was occurred and this agrees with the study of Walker's analysis [39] who believes that changes seen in the protraction mechanics and not from expansion appliance. 
We compared the treatment effects of a FM-BB protocol in Class III malocclusion and growth changes in untreated Class III subjects. That following treatment and post treatment craniofacial modifications were seen during an average observation period .Also, the present study showed non-significant changes in vertical measurements expect in inclination of palatal plane to the cranial base and FH to the mandibular plane (SN-PP, FH-MP) showed significant smaller value. In contrast to the results reported by Kersten et al. 1998 [40], these changes due to the effect of face mask and Bite Block that prevent the clock wise rotation of the mandible and palatal plane. That increased the relationship between the mandibular plane and palatal plane. However, these short term changes described previously by Deguchi et al. [41] disapproved by long term observation. Dental changes showed significant changes in maxillary and mandibular incisor inclination and over jet was significantly improved during active treatment from T2 to T1. This finding agrees with findings reported by Westwood et al., Chang et al. and Deguchi et al. [27] [37] [41]. However, this finding does not agree with results reported by Gozza et al. [38] and this is due to the use of an acrylic Bite Block in lower jaw which prevents retraction of lower incisors.

Finally, treatment during mixed dentition is preferable for many reasons, such as not to expose children to surgical procedures, rejection of parents to use any surgical techniques, and avoidance of any injury to still erupted permanent teeth. Consequently, from my clinical point of view, recently used methods, such as miniplates may be considered as invasive technique in mixed dentition for depending totally on surgical procedures. However, it is better to be used after complete eruption of all permanent teeth.

\section{Conclusions}

- Treatment with the FM-BB protocol induced significant dentoskeletal responses in terms of improvement of SNA angle, ANB angle, OJ, and molar relationship.

- Significant dentoskeletal changes in treated versus control group.

- Protraction perse produce significant changes from T1 to T2 without expansion.

- Maxillary acrylic splint with face mask significantly improved class III with retruded maxilla.

\section{References}

[1] Baccetti, T., McGill, J.S., Franchi, L., McNamara, J.A. and Tollaro, I. (1998) Skeletal Effects of Early Treatment of Class III Malocclusion with Maxillary Expansion and Face-Mask Therapy. American Journal of Orthodontics and Dentofacial Orthopedics, 113, 333-343. http://dx.doi.org/10.1016/S0889-5406(98)70306-3

[2] Cahng, H.F., Chen, K.C. and Nanda, R. (1997) Two-Stage Treatment of a Sever Skeletal Class III, Deep Bite Malocclusion. American Journal of Orthodontics and Dentofacial Orthopedics, 111, 481-486. http://dx.doi.org/10.1016/S0889-5406(97)70283-X

[3] Macdonald, K.E., Kapust, A.J. and Turley, P.K. (1999) Cephalometric Changes after the Correction of Class III Malocclusion with Maxillary Expansion/Face Mask Therapy. American Journal of Orthodontics and Dentofacial Orthopedics, 116, 13-24. http://dx.doi.org/10.1016/S0889-5406(99)70298-2

[4] Proffit, W.R. (1986) Contemporary Orthodontics. CV Mosby Company, St Louis, Toronto, London.

[5] Silva, O.G., Magro, A.C. and Capelozza, L. (1998) Early Treatment of Class III Malocclusion with Rapid Maxillary Expansion and Maxillary Protraction. American Journal of Orthodontics and Dentofacial Orthopedics, 113, $196-203$. http://dx.doi.org/10.1016/S0889-5406(98)70292-6

[6] Abdel-Ghany, A.H. (1995) The Effect of Protraction Face Mask on the Treatment of Maxillary Deficiency in Two Age Groups. Doctor Degree Thesis, Cairo University, Cairo.

[7] Alcan, T., Keles, A. and Erverdi, N. (2000) The Effects of a Modified Protraction and Head Gear on Maxilla. American Journal of Orthodontics and Dentofacial Orthopedics, 117, 27-38. http://dx.doi.org/10.1016/S0889-5406(00)70245-9

[8] Kulbersh, V.P., Berger, J. and Kersten, G. (1998) Effects of Protraction Mechanisms on the Midface. American Journal of Orthodontics and Dentofacial Orthopedics, 114, 489-491.

[9] Grattini, G., Levrini, L., Grozzoli, P. and Levrini, A. (1998) Skeletal and Dental Modifications Produced by the Bionator III Appliance Malocculusion Appliance. The American Journal of Orthopedics, 114, 4.

[10] Enacar, A. and Demrhanglu, M. (1989) Delaire Verdon Türü orthopedic yüz maskinin yapim Veuygulanmasinda pratik bir yontem. Türk ortodon Dergisi, 2, 183-188.

[11] Delaire, J. (1976) Le syndrome prognathique mandibulaire. Orthodontic Free Journal, 47, 203-219. 
[12] Fränkel, R. (1970) Maxillary Retrusion in Class III Malocclusion with the Function Corrector III. Trans Eur Orthod Soc, 46, 249-259.

[13] Tollaro, I., Baccetti, T. and Franchi, L. (1995) Mandibular Skeletal Changes Induced by Early Functional Treatment of Class III Malocclusion: A Superimposition Study. American Journal of Orthodontics and Dentofacial Orthopedics, 108, 525-532. http://dx.doi.org/10.1016/S0889-5406(95)70053-6

[14] Üçem, T.T., Ucuncu, N. and Yüksel, S. (2004) Comparison of Double-Plate Appliance and Facemask Therapy in Treating Class III Malocclusions. American Journal of Orthodontics and Dentofacial Orthopedics, 126, 672-679. http://dx.doi.org/10.1016/j.ajodo.2003.09.035

[15] Graber, L.W. (1977) Chin Cup Therapy for Mandibular Prognathism. American Journal of Orthodontics, $72,23-41$. http://dx.doi.org/10.1016/0002-9416(77)90122-1

[16] Ferro, A., Perillo Nucci, L., Ferro, F. and Gallo, C. (2003) Long-Term Stability of Skeletal Class III Patients Treated with Splints, Class III Elastics, and Chincup. American Journal of Orthodontics and Dentofacial Orthopedics, 123, 423-434. http://dx.doi.org/10.1067/mod.2003.70

[17] Cozzani, G. (1981) Extraoral Traction and Class III Treatment. American Journal of Orthodontics, 80, 638-650. http://dx.doi.org/10.1016/0002-9416(81)90266-9

[18] Rey, D., Angle, D., Oberti, G. and Baccetti, T. (2008) Treatment and Post Treatment Effects of Mandibular Cervical Headgear Followed by Fixed Appliances in III Malocclusion. American Journal of Orthodontics and Dentofacial Orthopedics, 133, 371-378. http://dx.doi.org/10.1016/j.ajodo.2006.04.043

[19] Irie, M. and Nakamura, S. (1975) Orthopedic Approach to Severe Skeletal Class III Malocclusion. American Journal of Orthodontics, 67, 377-392. http://dx.doi.org/10.1016/0002-9416(75)90020-2

[20] Delaire, J. (1997) Maxillary Development Revisited: Relevance to the Orthopaedic Treatment of Class III Malocclusions. European Journal of Orthodontics, 19, 289-311. http://dx.doi.org/10.1093/ejo/19.3.289

[21] Williams, M.D., Sarver, D.M., Sadowsky, P.L. and Bradley, F. (1997) Combined Rapid Maxillary Expansion and Protraction Facemask in the Treatment of Class III Malocclusions in Growing Children: A Prospective Long-Term Study. Seminars in Orthodontics, 3, 265-274. http://dx.doi.org/10.1016/S1073-8746(97)80059-X

[22] Hägg, U., Tse, A., Bendeus, M. and Rabie, A.B. (2003) Long-Term Follow-Up of Early Treatment with Reverse Headgear. European Journal of Orthodontics, 25, 95-102. http://dx.doi.org/10.1093/ejo/25.1.95

[23] Wells, A.P., Sarver, D.M. and Proffit, W.R. (2006) Long-Term Efficacy of Reverse Pulls Headgear Therapy. The Angle Orthodontist, 76, 915-922. http://dx.doi.org/10.2319/091605-328

[24] Kuster, R. and Ingervall, B. (1992) The Effects of Treatment of Skeletal Open Bite with Two Types of Bite-Blocks. European Journal of Orthodontics, 14, 489-499. http://dx.doi.org/10.1093/ejo/14.6.489

[25] McNamara, J.A. (1987) An Orthopedic Approach to the Treatment of Class III Malocclusion in Young Patients. Journal of Clinical Orthodontics, 21, 598-608.

[26] Baccetti, T., Franchi, L. and McNamara, J.A. (2000) Treatment and Post Treatment Craniofacial Changes after Rapid Maxillary Expansion and Facemask Therapy. American Journal of Orthodontics and Dentofacial Orthopedics, 118, 404-413. http://dx.doi.org/10.1067/mod.2000.109840

[27] Westwood, P.V., McNamara, J.A., Baccetti, T., Franchi, L. and Sarver, D.M. (2003) Long-Term Effects of Class III Treatment with Rapid Maxillary Expansion and Facemask Therapy Followed by Fixed Appliances. American Journal of Orthodontics and Dentofacial Orthopedics, 123, 306-320. http://dx.doi.org/10.1067/mod.2003.44

[28] McNamara, J.A. (1977) An Experimental Study of Increased Vertical Dimension in the Growing Face. American Journal of Orthodontics, 71, 382-395. http://dx.doi.org/10.1016/0002-9416(77)90242-1

[29] Jacobson, A. (1976) Application of the “Wits” Appraisal. American Journal of Orthodontics, 70, 179-189. http://dx.doi.org/10.1016/S0002-9416(76)90318-3

[30] Baccetti, T., Franchi, L. and McNamara, J.A. (2005) The Vertical Vertebral Maturation (CVM) Method for the Assessment of Optimal Treatment Timing in Dentofacial Orthopedics. Seminars in Orthodontics, 11, 119-129. http://dx.doi.org/10.1053/j.sodo.2005.04.005

[31] Ricketts, R.M. (1960) The Influence of Orthodontic Treatment on Facial Growth and Development. The Angle Orthodontist, 30, 103-133.

[32] Steiner, C.C. (1953) Cephalometrics for You and Me. American Journal of Orthodontics, 39, 729-755. http://dx.doi.org/10.1016/0002-9416(53)90082-7

[33] Tweed, C. (1966) Clinical Orthodontics. C.V. Mosby, St Louis.

[34] Shanker, S. (1996) Cephalometric A Point Changes during and after Maxillary Protraction and Expansion. American Journal of Orthodontics and Dentofacial Orthopedics, 110, 423-430.

http://dx.doi.org/10.1016/S0889-5406(96)70046-X 
[35] Kambara, T. (1977) Dentofacial Changes Produced by Extraoral Forward in Macaca irus. American Journal of Orthodontics, 71, 249-277. http://dx.doi.org/10.1016/0002-9416(77)90187-7

[36] Nanda, R. (1978) Protraction of Maxilla in Rhesus Monkeys by Controlled Extra Oral Forces. American Journal of Orthodontics, 74, 121-141. http://dx.doi.org/10.1016/0002-9416(78)90080-5

[37] Chang, H.-P., Kinoshita, Z. and Kawamoto, T. (1992) Craniofacial Pattern of Class III Deciduous Dentition. The Angle Orthodontist, 62, 139-144.

[38] Cozza, P., Marino, A. and Mucedero, M. (2004) An Orthopaedic Approach to the Treatment of Class III Malocclusions in the Early Mixed Dentition. European Journal of Orthodontics, 26, 191-199. http://dx.doi.org/10.1093/ejo/26.2.191

[39] Leonard, M. and Walker, G.F. (1977) A Cephalometric Guide to the Diagnosis of Midface Hypoplasia at the Le Fort II Level. Journal of Oral Surgery, 35, 21-24.

[40] Pangrazio-Kulbersh, V., Berger, J. and Kersten, G. (1998) Effects of Protraction Mechanics on the Midface. American Journal of Orthodontics and Dentofacial Orthopedics, 114, 484-491. http://dx.doi.org/10.1016/S0889-5406(98)70167-2

[41] Mimura, H. and Deguchi, T. (1996) Morphologic Adaptation of Temporomandibular Joint after Chincup Therapy. American Journal of Orthodontics and Dentofacial Orthopedics, 110, 541-546. http://dx.doi.org/10.1016/S0889-5406(96)70063-X 
Scientific Research Publishing (SCIRP) is one of the largest Open Access journal publishers. It is currently publishing more than 200 open access, online, peer-reviewed journals covering a wide range of academic disciplines. SCIRP serves the worldwide academic communities and contributes to the progress and application of science with its publication.

Other selected journals from SCIRP are listed as below. Submit your manuscript to us via either submit@scirp.org or Online Submission Portal.
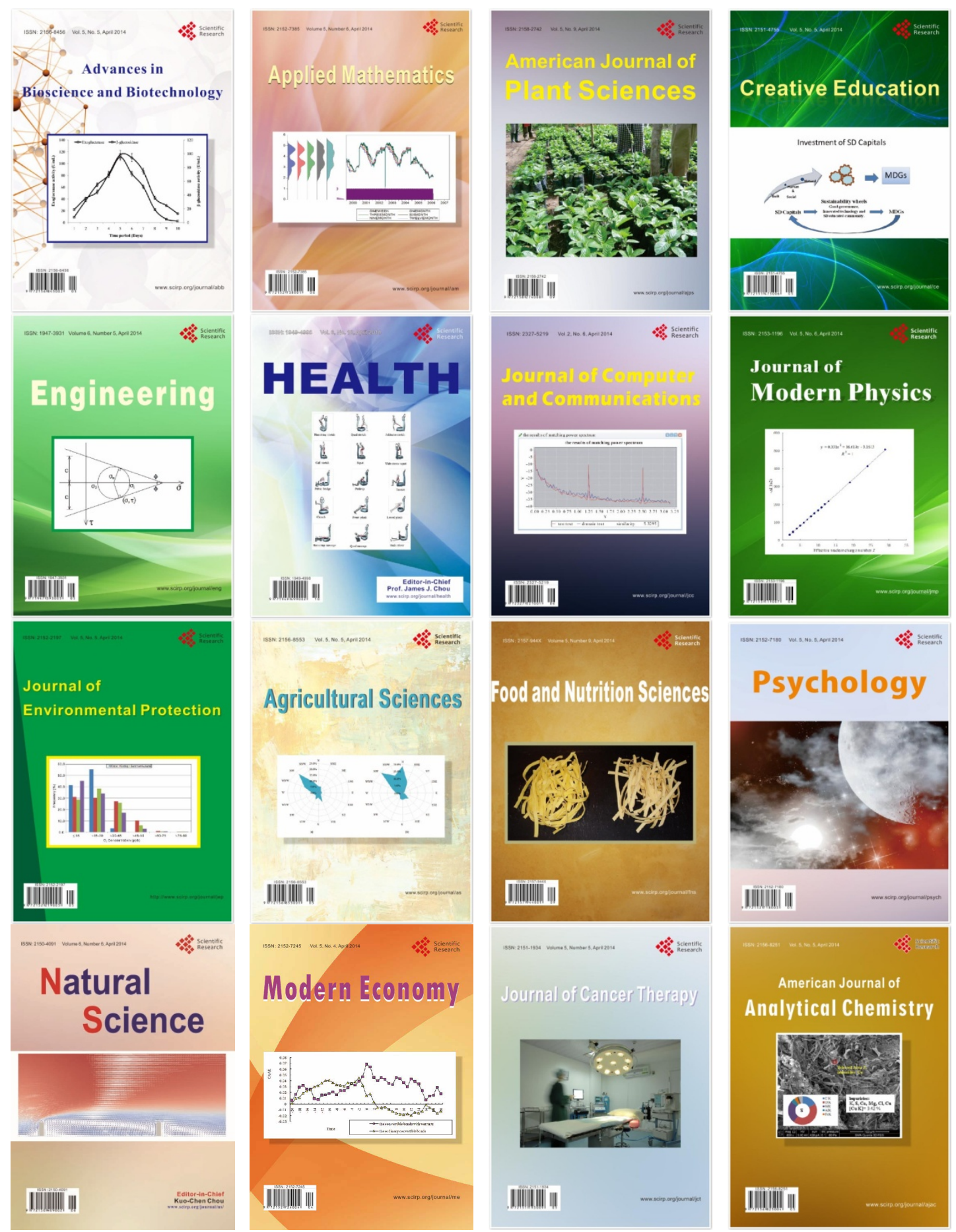